\title{
Scalp Psoriasiform Contact Dermatitis with Acute Telogen Effluvium due to Topical Minoxidil Treatment
}

\author{
Michelangelo La Placa Riccardo Balestri Federico Bardazzi

\section{Colombina Vincenzi} \\ Dermatology Division, Department of Experimental, Diagnostic and Specialty Medicine, University of Bologna, \\ Bologna, Italy
}

\section{Established Facts}

- Allergic contact dermatitis (ACD) from minoxidil solution is well known, and several cases have been reported.

- Scalp ACD may be the cause of telogen effluvium (TE), and patch tests are warranted for diagnosis.

\section{Novel Insight}

- We describe 2 cases of psoriasiform ACD of the scalp and severe TE due to minoxidil treatment.

\section{Key Words}

Minoxidil · Contact dermatitis · Psoriasis · Telogen

effluvium · Scalp $\cdot$ Hair loss $\cdot$ Propylene glycol

\begin{abstract}
Topical minoxidil, the only approved treatment for female pattern hair loss (FPHL), has been associated with scalp allergic contact dermatitis (ACD). We report the case of $2 \mathrm{fe}$ male patients who developed ACD from minoxidil solution with severe telogen effluvium and psoriasiform scalp dermatitis. Scalp dermoscopy was useful to identify the psoriasiform vascular pattern, whereas patch testing made it possible to differentiate the cause of sensitization. In one case,
\end{abstract}

minoxidil was the sole cause of scalp dermatitis, while in the other patient it was only the vehicle, thus permitting the patient to continue the treatment for FPHL.

(c) 2015 S. Karger AG, Base

\section{Introduction}

Topical minoxidil is the only approved treatment for female pattern hair loss (FPHL), and its use is effective in most women for the stabilization of hair loss and/or hair regrowth. Mild pruritus or scaling may be experienced during the first 1-2 months of treatment due to the fact that minoxidil induces fast reentry in the anagen of the

\section{KARGER 125}

C 2015 S. Karger AG, Basel

2296-9195/15/0013-0141\$39.50/0

E-Mail karger@karger.com

www.karger.com/sad
Colombina Vincenzi, Dermatology Division

Department of Experimental, Diagnostic and Specialty Medicine University of Bologna, Via Massarenti 1 IT-40138 Bologna (Italy)

E-Mail colombina.vincenzi@ unibo.it 
Fig. 1. a Diffuse hair loss with severe scaling and scalp erythema. b Scalp dermoscopy of patient 1 , showing numerous scales and vascular twisted loops.
Fig. 2. a The patch test results in patient 1 shows a positive reaction to her minoxidil solution and a negative reaction to propylene glycol. $\mathbf{b}$ The patch test results in patient 2 reveals a positive reaction to both her minoxidil solution and propylene glycol.
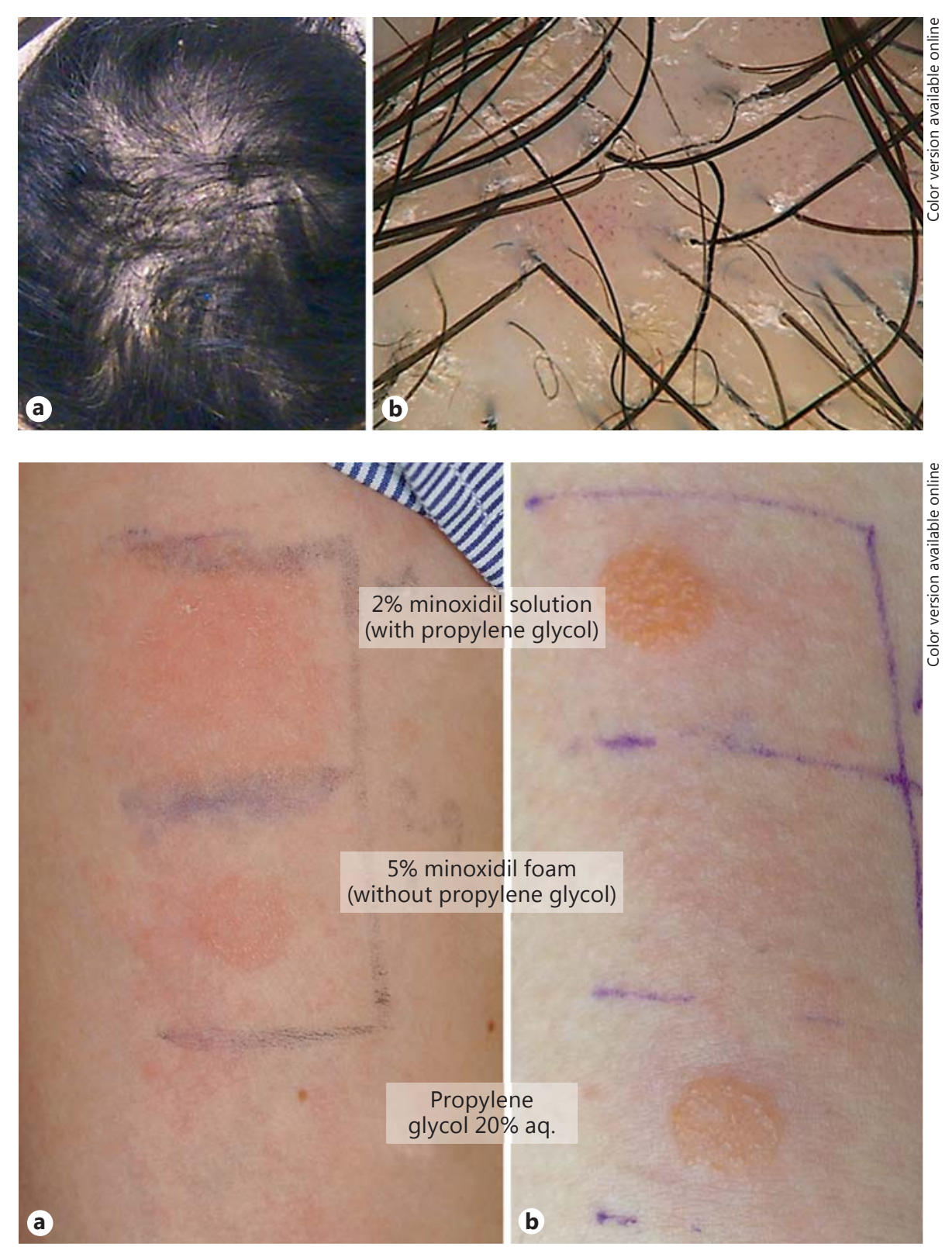

hair follicles, provoking a transitory telogen effluvium (TE). If symptoms persist or appear later during the treatment, the occurrence of sensitization to minoxidil or to the vehicles must be excluded in order to avoid an acute TE that could compromise the benefits of minoxidil. Allergic contact dermatitis (ACD) from minoxidil solution is well known, and several cases have been reported where either minoxidil or propylene glycol have been identified as the cause of allergy $[1,2]$.

We herein report 2 cases of psoriasiform ACD of the scalp and acute TE due to minoxidil solution allergy.

\section{Case Presentations}

Case 1

A 46-year-old woman with severe FPLH had been under treatment with $2 \%$ topical minoxidil (Regaine ${ }^{\circledR}$ ) for several years. The patient was referred to our consultation due to her complaining of a deterioration of her symptoms. We observed diffused alopecia, with large areas of scaly psoriatic plaques involving almost the entire scalp, extending from the frontal hairline to the vertex (fig. 1a). The pull test was strongly positive, and dermoscopic evaluations (fig. 1b) confirmed the presence of a psoriasiform vascular pattern characterized by twisted hair loops. According to the patient, the hair loss had worsened over the last few weeks in association with 
the abrupt appearance of scaly dermatosis. Laboratory investigations were within the normal range.

Patch tests performed with her personal $2 \%$ minoxidil solution (containing propylene glycol as eccipient), with a 5\% minoxidil foam without propylene glycol (Rogaine ${ }^{\circledR}$ foam for men) and with propylene glycol $20 \%$ aq. (Allergopharma S.p.A., Rome, Italy) revealed a positive reaction $(2+)$ to the personal $2 \%$ minoxidil solution and to the $5 \%$ minoxidil foam at day 4 , with a negative reaction to propylene glycol (fig. 2a). Minoxidil treatment was subsequently discontinued, and topical therapy with clobetasol ointment under occlusion was started. The psoriasiform dermatitis and TE improved within a few weeks.

Case 2

A 28-year-old otherwise healthy female was seen because of acute diffuse hair loss. She had a 1-year history of FPHL under treatment with $2 \%$ topical minoxidil solution. Routine blood tests were within the normal limits. Clinical observation and a positive pull test suggested the diagnosis of TE. Dermoscopic evaluation showed the presence of numerous scales associated with the typical psoriatic vascular pattern with twisted loops.

At day 4, patch tests revealed a strong positive reaction $(2+)$ to her personal $2 \%$ minoxidil solution and to propylene glycol, but a negative reaction to the $5 \%$ minoxidil foam (fig. $2 \mathrm{~b}$ ). In this patient, propylene glycol, and not minoxidil itself, was likely to be the cause of sensitization. After complete healing from the psoriasiform dermatitis, $5 \%$ minoxidil foam without propylene glycol was prescribed.

\section{Discussion}

TE is a very distressing condition, characterized by increased hair shedding, potentially reversible, without complete hair loss, usually starting a few months after a triggering factor, including psychoemotional stress, imbalanced diet and drug intake. Several cases of TE occurring after an episode of ACD to hair dyes have been previously described, provoking diffused nonscarring alopecia in these patients [3].
The acute flare-up of psoriasis is possible after any trauma, including burns, insect bites, tattooing and surgery, and occurs in about $25 \%$ of the psoriatic individuals under the name of the Koebner phenomenon [4]. However, it has never been reported after ACD so far.

We described 2 female patients under treatment with minoxidil solution for FPHL worsened by the contemporaneous presence of scalp psoriasiform ACD and TE. To the best of our knowledge, this is the first report describing an acute TE after ACD from minoxidil.

In our patients, the abrupt flare-up of psoriasiform ACD and acute TE were the main cause of concern and the reason for seeking our medical advice. Scalp dermoscopy was useful to identify the psoriasiform vascular pattern [5], and patch tests made it possible to differentiate the cause of sensitization in these 2 cases. In case 1 , minoxidil was the sole cause of scalp dermatitis, while in case 2 it was only the vehicle, thus permitting us to continue the treatment for FPHL.

Although without a scalp biopsy it is impossible to definitely establish whether it is psoriasis koebnerized by contact allergy or a psoriasiform allergic reaction, it is important for the dermatologist to recognize that a minoxidil treatment allergy may be the cause of scalp psoriasiform ACD followed by acute TE with severe hair loss, and that patch tests are warranted for diagnosis.

\section{Statement of Ethics}

Consent was obtained from the patients.

\section{Disclosure Statement}

There are no conflicts of interest to disclose.

\section{References}

Psoriasiform Allergic Contact Dermatitis from Minoxidil
1 Hagemann T, Schlütter-Böhmer B, Allam JP, et al: Positive lymphocyte transformation test in a patient with allergic contact dermatitis of the scalp after short-term use of topical minoxidil solution. Contact Dermatitis 2005;33: 53-55.

2 Friedman ES, Friedman PM, Cohen DE, et al: Allergic contact dermatitis to topical minoxidil solution: etiology and treatment. J Am Acad Dermatol 2002;46:309-312.
3 Tosti A, Piraccini BM, van Neste DJJ: Telogen effluvium after allergic contact dermatitis of the scalp. Arch Dermatol 2001;137:187-190.

4 Sagi L, Trau H: The Koebner phenomenon. Clin Dermatol 2011;29:231-236.

5 Ross EK, Vincenzi C, Tosti A: Videodermoscopy in the evaluation of hair and scalp disorders. J Am Acad Dermatol 2006;55:799-806. 